\title{
Nuclear magnetic resonance imaging of absorption of electromagnetic radiation in brain tissue
}

\section{David H. Gültekin}

Memorial Sloan-Kettering Cancer Center, Weill Cornell Medical College, New York, USA

\section{ARTICLE INFO}

\section{* Correspondence to:}

David H. Gültekin

Memorial Sloan-Kettering Cancer Center,

Weill Cornell Medical College,

New York, USA

e-mail: david.gultekin@aya.yale.edu

\section{Keywords:}

Nuclear magnetic resonance imaging Brain

Radio frequency

Specific absorption rate

\section{ABSTRACT}

Nuclear magnetic resonance (NMR) imaging is effectively used for measuring the absorption of electromagnetic radiation in the brain tissue noninvasively. The absorption of electromagnetic energy in the brain transforms into thermal energy and affects the nuclear magnetic shielding of the molecules. The subsequent temperature increase caused by the absorption of energy is then measured by the resonance frequency shift of hydrogen protons of the water molecules in the brain tissue. NMR imaging provides fast three dimensional (3D) distribution of thermal energy in the brain during the exposure to power and time varying radio frequency (RF) fields. A method is presented for measuring the absorption of electromagnetic energy in ex vivo brain tissue subjected to a radiation from a half wavelength dipole antenna assembly. This method provides high spatial and temporal resolution to characterize the hot spots formed by the absorption of cell phone radiation in brain tissue and allows for the measurements of the specific absorption rate averaged over $1 \mathrm{mg}$ and $10 \mathrm{~s}$ in the brain tissue throughout the total absorption volume. NMR thermometry and fiber optic temperature sensors confirm the accuracy of the measurements.

J.Exp.Clin.Med., 2013; 30: 269 